\title{
Peripheral capillary non-perfusion in treatment-naïve proliferative diabetic retinopathy associates with postoperative disease activity 6 months after panretinal photocoagulation
}

\author{
Thomas Lee Torp, ${ }^{1,2}$ Ryo Kawasaki, ${ }^{3}$ Tien Yin Wong, ${ }^{4}$ Tunde Peto, ${ }^{2,5}$ Jakob Grauslund ${ }^{1,2}$
}

\begin{abstract}
'Department of Ophthalmology, Odense University Hospital, Odense, Denmark

${ }^{2}$ Department of Clinical Research, University of Southern Denmark, Odense, Denmark ${ }^{3}$ Department of Vision Informatics, Osaka University Graduate School of Medicine, Osaka, Japan

${ }^{4}$ Singapore National Eye Centre, Duke-NUS Medical School, National University of Singapore, Singapore ${ }^{5}$ School of Medicine, Dentistry and Biomedical Sciences, Queen's University Belfast, Belfast, UK
\end{abstract}

\section{Correspondence to} Dr Thomas Lee Torp, Department of Ophthalmology, Odense University Hospital, Odense 5000, Denmark; thomas.lee. torp@rsyd.dk

Received 11 March 2018 Revised 6 June 2018 Accepted 2 July 2018 Published Online First 1 August 2018
ABSTRACT

Background/aims With the perspective to provide individualised panretinal laser photocoagulation (PRP) for proliferative diabetic retinopathy (PDR), we evaluated if retinal peripheral capillary non-perfusion (PCNP) and oximetry, as non-invasive markers of retinal metabolism and function, could predict disease activity 6 months after PRP.

Methods We performed a prospective, interventional study of patients with treatment-naïve PDR. Retinal oximetry and ultra-widefield fluorescein angiography were performed at baseline (BL) and three (3M) and 6 months (6M) after PRP by a navigated laser system. At $6 \mathrm{M}$ follow-up, patients were divided according to disease activity: active or inactive.

Results We included 33 eyes, and $69.6 \%$ were men. At $\mathrm{BL}$, the median age and duration of diabetes (with IQRs) were $51.6 \pm 23.4$ and $20.0 \pm 15.0$ years. Haemoglobin $A_{1 c}$ was $63.0 \pm 17.0 \mathrm{mmol} / \mathrm{mol}$ and blood pressure was $152 \pm 37 / 82 \pm 24 \mathrm{~mm} \mathrm{Hg}$. At BL and M6, patients with postoperative disease activity $(30.3 . \%, n=10)$ had a larger area with PCNP than those with inactive PDR (BL: $51 \%-75 \%$ vs $26 \%-50 \%$, p=0.03; 6M: $51 \%-75 \%$ vs $26 \%-50 \%, p=0.03$ ). The area of PCNP did not change from $B L$ to $6 \mathrm{M}$ in either group (inactive PDR: $p=0.38$, active PDR: $p=0.87$ ). Changes in retinal oxygen saturation were not found to be clinical relevant.

Conclusion We found the area of PCNP at all timepoints to be statistically larger in patients with active PDR 6 months after PRP treatment. Therefore, the area of PCNP, at baseline, may serve as a potential predictive marker for PDR activity after treatment.

\section{INTRODUCTION}

Diabetic retinopathy (DR) is a microvascular disease induced by metabolic disturbances in the retinal homeostasis leading to retinal hypoxia. ${ }^{1}$ Proliferative diabetic retinopathy (PDR) is a late complication, which remains an important cause of severe visual loss around the world. ${ }^{2}{ }^{3}$ PDR is characterised by the formation of retinal neovascularisations, potentially resulting in vitreous haemorrhage or tractional retinal detachment. ${ }^{4}$ Panretinal laser photocoagulation (PRP) halves the risk of severe visual loss as demonstrated by the DRS study in the late 1970 s. $^{5}$ Since then, PRP has been the gold standard of treatment for PDR. Nevertheless, post-laser activity of PDR can be hard to predict, and some patients may require more PRP (number of treatment episodes and number of burns) than others. Non-invasive preoperative markers will need to provide more precise PRP treatment algorithms, optimising the balance between treatment efficacy and side effects like visual field loss, night blindness and diabetic macular oedema.

The vast majority of the retinal area is located outside the traditional seven fields of the ETDRS. The retinal periphery has important prognostic value when evaluating disease activity, ${ }^{67}$ and it has been demonstrated that the presence of peripheral retinal diabetic lesions are correlated with a 4.7-fold increase risk of developing PDR. ${ }^{8}$ Furthermore, the overall hypoxic state of the retina is driven by capillary occlusion and retinal non-perfusion, which is predominantly found in the retinal periphery in association with severe DR. ${ }^{8-10}$ Peripheral capillary non-perfusion (PCNP) can be assessed by ultra-widefield fluorescein angiography (UWFA) covering a 200-degree overview of the retina, as compared with the conventional image of 30-50 degrees. Retinal oximetry is another promising marker for retinal metabolism; several studies demonstrated increased retinal venular oxygen saturation in association with increased levels of DR. ${ }^{11-15}$ These non-invasive measurements of retinal function and metabolism may serve as risk factors for disease activity. To determine this, we aimed to test if PCNP and oxygen saturation could predict disease activity 6 months (6M) after PRP in treatment-naïve patients with PDR.

\section{MATERIALS AND METHODS}

We carried out a $6 \mathrm{M}$ prospective, interventional study of 33 treatment-naïve eyes with PDR treated at Odense University Hospital, Denmark, between August 2014 and October 2015. We excluded patients with clinical significant macular oedema, previous PRP and treatment-requiring cataract, age $<18$ years or those pregnant. We only included one eye from each patient in order to avoid possible intereye correlations. ${ }^{16}$

The study was registered at the Regional Scientific Ethics Committee (ID S-20140046), the Danish Data Protection Agency (ID 14/16546), ClinicalTrials.gov (ID NCT02157350) and conducted in accordance with the criteria of Helsinki II Declaration and good clinical practice. The patient sample presented in this study is part of a bigger cohort, and data regarding retinal oxygen saturation and vascular geometry have previously been published. ${ }^{17} 18$ 


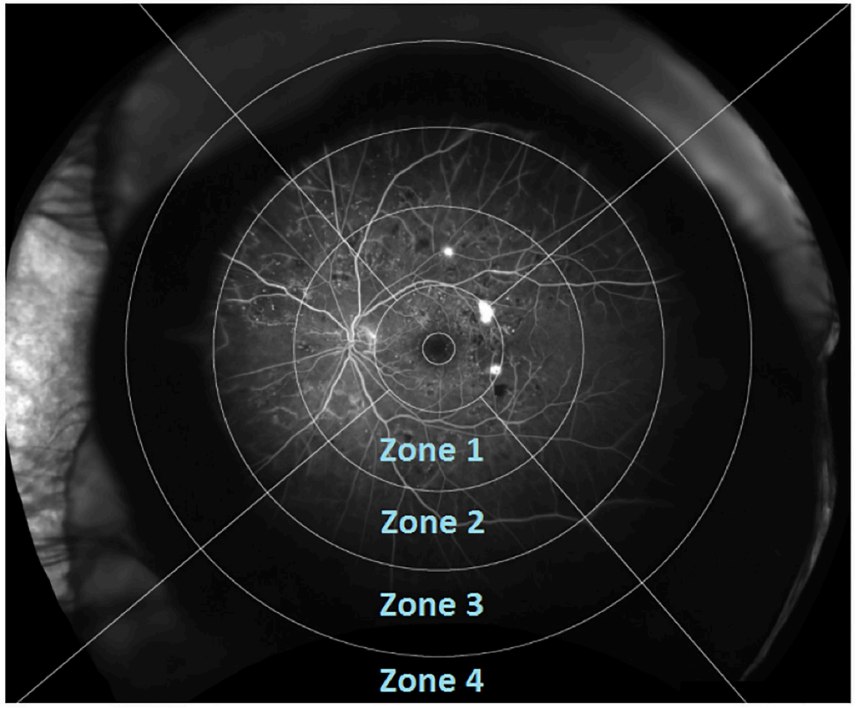

Figure 1 Ultra-widefield fluorescein angiography (OPTOS) of left eye with overlaid grading grid dividing the retinal into 12 zones, each graded according to area of capillary non-perfusion (CNP) (no CNP, $1 \%-25 \%, 26 \%-50 \%, 51 \%-75 \%, 76 \%-100 \%$ or ungradable).

\section{Baseline examinations}

All patients provided a full medical history and underwent a thorough eye examination including mydriatic (tropicamide 10 $\mathrm{mg} / \mathrm{mL}$ and phenylephrine 10\%) slit-lamp examination, intraocular pressure (Goldmann applanation tonometry), optical coherence tomography (OCT) by 3D OCT-2000 Spectral domain OCT (Topcon, Tokyo, Japan), retinal oximetry (Oxymap model T1; Oxymap, software V.2.5.1, Reykjavik, Iceland) and blood pressure on the upper arm after $10 \mathrm{~min}$ in rest (Omron $705 \mathrm{CP}$, Hoofdrop, The Netherlands), and Haemoglobin $\mathrm{A}_{1 \mathrm{c}}$ (HbA1c) was analysed centrally by wash solution resin photometry, ion exchange HPLC-UV (Tosoh G8; normal range $<48 \mathrm{mmol} / \mathrm{mol}$ ). The baseline examinations were supplemented by UWFA and imaging (Optomap; OPTOS 200Tx, Optos PLC, Dunfermline,
Scotland, UK), and the diagnosis of PDR was verified by a retinal specialist (JG) before the patient was included in the study.

PRP was given in two sessions by a navigated laser system (NAVILAS; OD-OS GmbH, Berlin, Germany) with a NAVILAS $34 \mathrm{~mm}$ or $38 \mathrm{~mm}$ contact lens in local anaesthesia (oxybuprocain hydrochloride $0.4 \%$ ). All treatments were given by certified personnel (TLT and JG), and a treatment summary was automatically provided by the laser system after each session.

\section{Follow-up examinations}

Follow-up examinations were performed 6M after PRP, and baseline (BL) examinations were repeated. At $6 \mathrm{M}$, patients were divided into two groups depending on the activity of PDR: active or inactive PDR. Active PDR was defined as either the formation of new retinal proliferations, or increased area of one or more proliferations, or increased area of fluorescein leakage as given by UWFA. Patients were also seen 3 months (3M) after PRP, and rescue laser treatment was given if indicated $(n=2)$.

\section{Retinal peripheral capillary non-perfusion}

The retinal areas of PCNP were assessed by UWFA images $1 \mathrm{~min}$ after fluorescein injection, and a macular centred grading grid was placed on the image using a Matlab add-on program. ${ }^{19}$ The grader was allowed to adjust brightness/contrast when grading the different zones.

The grading grid divided the retinal UWFA image into 12 different zones, four in each retinal quadrant (superior, temporal, inferior and nasal) (figure 1). Zones were numbered 1 (innermost) through 4 (outermost). Each zone was manually categorised (graded) depending on the area of PCNP: 0 (no PCNP), 1 (1-25\% PCNP), 2 (26-50\% PCNP), 3 (51-75\% PCNP), 4 (76-100\% PCNP) or 5 (ungradable) (figure 2). Given that zones 1-4 differed in size, that the superior zone 4 was often ungradable due to eyelid interference and that zone 1 usually contained hyperfluorescent NVE, we used zone 3 as a marker for PCNP. The overall capillary density decreases towards the periphery of the retina, and thereby the difference in zone size was less important. But in order to get a global score that was

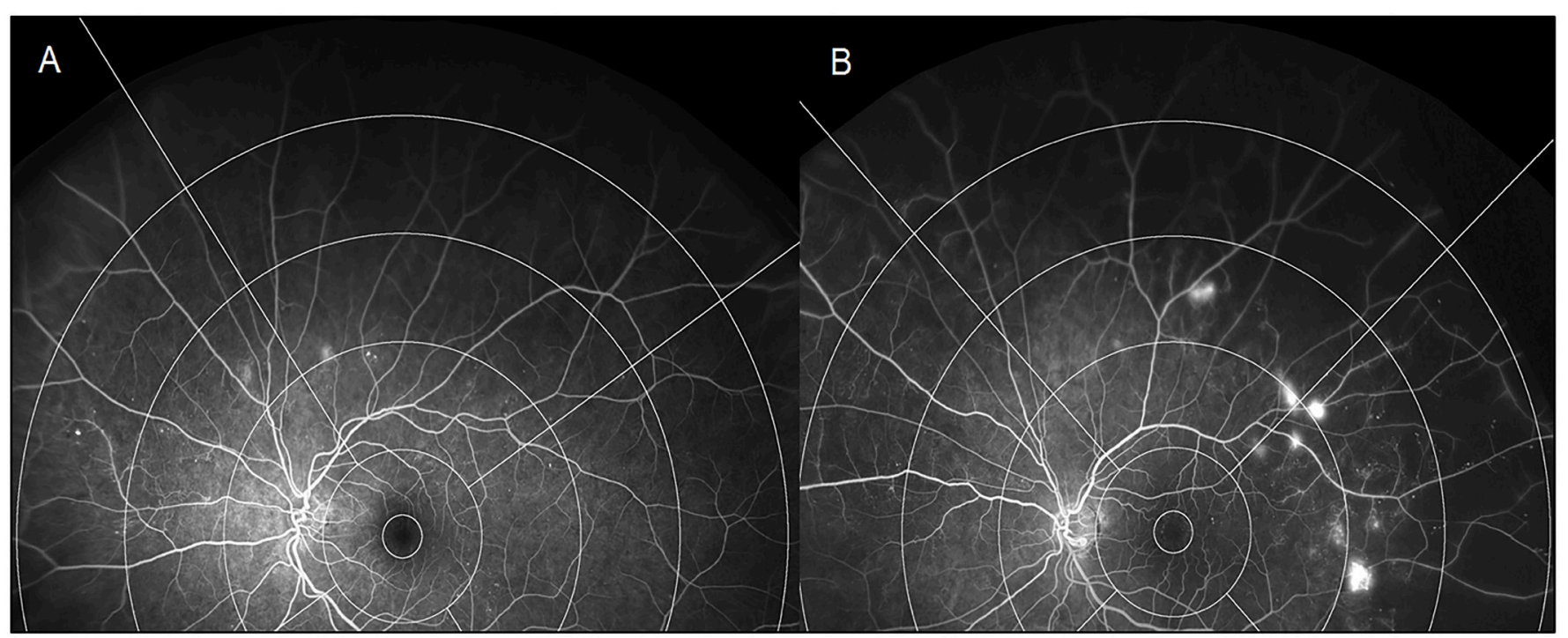

Figure 2 Baseline ultra-widefield fluorescein angiography (OPTOS) of patients with treatment-naïve proliferative diabetic retinopathy. (A) Patient with subsequent disease inactivity 6 months after panretinal photocoagulation (PRP). Zone 3 graded as no capillary non-perfusion. (B) Patient with subsequent disease inactivity 6 months after PRP. Zone 3 graded as 76\%-100\% capillary non-perfusion. 


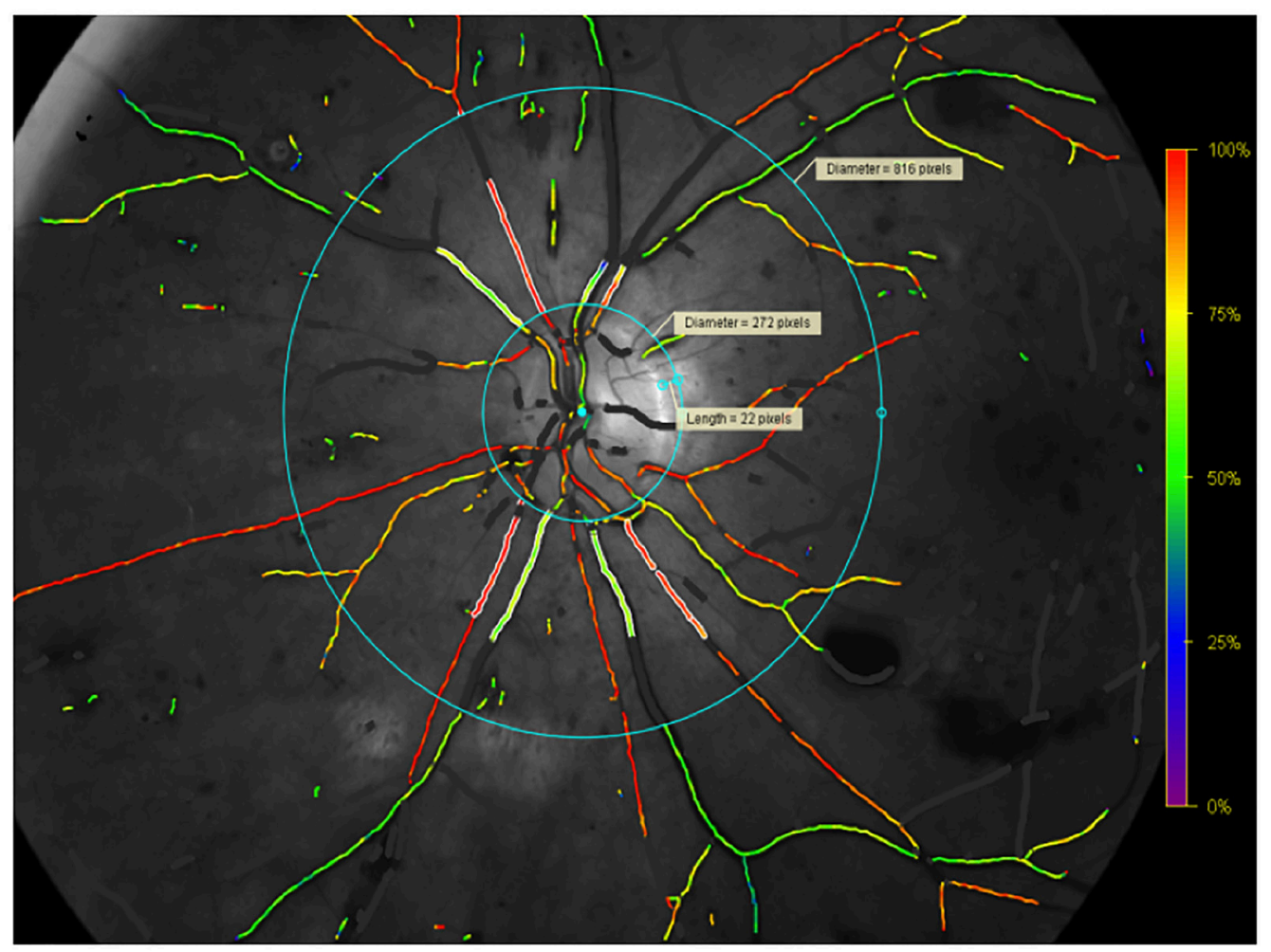

Figure 3 Oxymap T1 image showing traced vessels with overlaying colour map indicating oxygen saturation by colour in the left eye. Between the blue rings, the oxygen saturation is measured in traced vessels, seen as white lines on either side of the vessels. Retinal vessels covered in white areas are excluded vessel segments.

as uniform as possible, the global PCNP score was assessed by averaging the four equally sized quadrants of zone 3 . When evaluating the global PCNP score (continuous data), the focal grading scale (categorical) could not be used. Therefore, we used a moderated scale in order to assess the global PCNP score properly. The global PCNP score was evaluated according to the following moderated scale: $0-0.5$ (close to no CNP), >0.5-1.5 (1\%-25\%), >1.5-2.5 (26\%-50\%), >2.5-3.5 (51\%-75\%) and $>3.5-4.0(76 \%-100 \%)$.

\section{Retinal oxygen saturation}

Retinal oxygen saturation was performed using the Oxymap T1, with the procedure described previously. ${ }^{20} 21$ In brief, one 50-degree optic disc centred image was taken, with a flash intensity of 50 . Two rings were semiautomatically placed on the image. The inner ring was manually placed around the optic disc, with an antireflective margin of 20-30 pixels from the edge of the optic and outwards to the ring. The outer ring was automatically placed three disc diameters from the inner ring. One larger arteriole and venule in each quadrant was measured, tracing from the inner ring not exceeding the outer ring (figure 3 ). The vessel length had to be between 50 and 200 pixels. If the first branching point occurred before the vessel reached 50 pixels in length, the first branch vessel was chosen for measurement. All measurements were done on the same vessel segment at each time point. In order to assess the global retinal oxygen saturation, the mean retinal arteriolar and venular oxygen saturation of all four quadrants was used.

\section{Statistical analyses}

All statistical calculations were performed using STATA V.14.2 (StataCorp, College Station, Texas, USA), and $\mathrm{p}$ values under
0.05 were considered statistically significant. Categorical data are presented as percentage and continuous data as median with IQR. Differences between patients with inactive and active PDR were compared by the Mann-Whitney U test for continuous data and $\chi^{2}$ test for categorical data. The correlation between retinal oxygen saturation and PCNP were tested using the non-parametric test of Cuzick, and specific group comparisons were made by usage of the Mann-Whitney U test. Twenty per cent $(n=21)$ of the UWFA images were re-graded and showed a substantial intragrader agreement on areas of PCNP in zone $3(\mathrm{kappa}=0.66) .{ }^{22}$

\section{RESULTS}

We examined 33 eyes, and of those $69.6 \%$ were men and $21.2 \%$ were active smokers. At baseline, the median with IQR age and duration of diabetes were $51.6 \pm 23.4$ and $20.0 \pm 15.0$ years, respectively. $\mathrm{HbA} 1 \mathrm{c}$ was $63.0 \pm 17.0 \mathrm{mmol} / \mathrm{mol}$ and the blood pressure was $152 \pm 37 / 82 \pm 24 \mathrm{~mm} \mathrm{Hg}$. The global PCNP was $51 \%-75 \%(2.3)$, and the retinal global arteriolar and venular oxygen saturation was $95.7 \% \pm 6.9 \%$ and $67.2 \% \pm 13.4 \%$, respectively. At $6 \mathrm{M}$ follow-up, $30.3 \%(\mathrm{n}=10)$ had active PDR. At BL, patients who had inactive PDR and active PDR at $6 \mathrm{M}$ did not differ according to BL sex, smoking, age, duration of diabetes, HbA1c, blood pressure, laser energy delivered at baseline or retinal oximetry image quality (table 1). Furthermore, we found no correlation between retinal oxygen saturation and the area of PCNP at any time points when performing a test for trend across ordered groups (data not shown).

\section{Baseline}

At BL, patients with active PDR at $6 \mathrm{M}$ were found to have a significantly larger area of PCNP than those with inactive PDR $(51 \%-75 \%(3.3 \pm 1.3)$ vs 26\%-50\% $(2.0 \pm 2.3), \mathrm{p}=0.03)$ 
Table 1 Baseline characteristics according to activity of proliferative diabetic retinopathy 6 months after panretinal laser photocoagulation

\begin{tabular}{|c|c|c|c|}
\hline & Inactive PDR & Active PDR & \multirow[b]{2}{*}{$P$ values } \\
\hline & Median \pm IQR & Median \pm IQR & \\
\hline $\mathrm{n}$ & 23 & 10 & \\
\hline Sex, \% male & 78.3 & 50.0 & 0.10 \\
\hline Active smoking, \% & 26.0 & 10.0 & 0.29 \\
\hline Age, years & $50.9 \pm 23.4$ & $57.1 \pm 29.5$ & 0.50 \\
\hline Duration of diabetes, years & $18.0 \pm 17.0$ & $24.0 \pm 10.0$ & 0.23 \\
\hline $\mathrm{HbA} 1 \mathrm{c}, \mathrm{mmol} / \mathrm{mol}$ & $63.0 \pm 15.0$ & $74.5 \pm 22.0$ & 0.17 \\
\hline Systolic blood pressure, $\mathrm{mm} \mathrm{Hg}$ & $154 \pm 39$ & $149 \pm 46$ & 0.23 \\
\hline Diastolic blood pressure, $\mathrm{mm} \mathrm{Hg}$ & $82 \pm 25$ & $84 \pm 13$ & 0.96 \\
\hline Laser power, J & $6.70 \pm 2.54$ & $5.47 \pm 1.70$ & 0.34 \\
\hline 0ximetry image quality, $0-10$ & $8.1 \pm 1.0$ & $8.0 \pm 1.0$ & 0.82 \\
\hline
\end{tabular}

Continuous data are presented as median with IQR. Differences between groups were compared with the Mann-Whitney $\mathrm{U}$ test for continuous data and $\chi^{2}$ test for categorical. Oximetry image quality ranged from $0=$ poor to $10=$ excellent. PDR, proliferative diabetic retinopathy.

(table 2). No differences were found in retinal oxygen saturation between the two groups.

\section{Six months' follow-up}

At $6 \mathrm{M}$, similar to $\mathrm{BL}$, patients with active PDR had a larger area of PCNP as compared with patients with inactive PDR (51\%$75 \%(2.9 \pm 1.3)$ vs $26 \%-50 \%(2.0 \pm 1.6), \mathrm{p}=0.03)$ (table 2$)$. Moreover, when performing a Wilcoxon matched-pairs test on patients with active PDR, we found a statistically significant decrease in retinal arteriolar oxygen saturation from BL to $6 \mathrm{M}$ $(93.4 \% \pm 6.4 \%$ vs $93.3 \pm 7.2 \%, p=0.02)$.

\section{DISCUSSION}

In this prospective, interventional study, we found patients with active PDR 6M after PRP to have a larger area of PCNP at baseline and $6 \mathrm{M}$, as compared with patients with inactive PDR. Furthermore, it was found that increasing retinal vascular arteriolar oxygen saturation after PRP trended towards a lower risk of active PDR $(p=0.05)$, although patients with active PDR was found to decrease in retinal arteriolar oxygen saturation from BL to $6 \mathrm{M}$.

Earlier studies have shown that the retinal areas of non-perfusion increased with increased severity of $\mathrm{DR},{ }^{23}$ and that it plateaus at the stage of severe non-PDR. ${ }^{9}$ In our study, all patients (who by protocol had treatment-naïve PDR) had retinal areas of CNP at baseline. Furthermore, at BL, patients with active PDR had significantly larger areas of PCNP than patients with inactive PDR. This would indicate that patients with a lower retinal hypoxic load received sufficient PRP, while the same amount of treatment could be insufficient in patients with a higher hypoxic load. A successful response to the PRP treatment is not fully understood, but one hypothesis is that the retinal laser scars has a positive effect on the haemodynamic mechanisms of the retinal circulation, thereby reducing the hypoxic load and preventing progression of PDR. ${ }^{24}$

We found the same difference in area of PCNP between the two groups at $6 \mathrm{M}$, as found at BL. After PRP treatment, patients with active PDR were still seen to have larger areas of PCNP as compared with patients with inactive PDR. Traditionally, CNP is regarded an irreversible feature of $\mathrm{DR},{ }^{25}$ but reperfusion of occluded retinal capillary beds has been reported. ${ }^{26}$ The results found in our study did not show expansion in areas of PCNP in patients with active PDR or reduction in areas of PCNP in patients with inactive PDR from BL to $6 \mathrm{M}$. Optical coherence tomography angiography (OCT-A) is a novel non-invasive technique for imaging the microvasculature of the retina and choroid by detection of moving erythrocytes through different retinal segments . OCT-A would benefit greatly in trying to quantify the amount of retinal PCNP and might confirm the finding of this study. OCT-A has for the past couple of years advanced from 50-degree fundus images to widefield images, although the technique remains subject to known imaging complications such as retinal variations in illumination and artefacts.

The retinal oxygen saturation has in the literature been reported to be affected by PRP treatment. Conflicting changes

Table 2 Retinal oximetry and peripheral capillary non-perfusion results at baseline and 6 months follow-up.

\begin{tabular}{|c|c|c|c|c|c|}
\hline & \multicolumn{2}{|c|}{ Baseline } & \multirow{2}{*}{$\begin{array}{l}\text { 6M follow-up } \\
\text { Median } \pm I Q R\end{array}$} & \multirow{2}{*}{$\begin{array}{l}\text { Baseline-6M difference } \\
\text { Median } \pm \text { IQR }\end{array}$} & \multirow{2}{*}{$\begin{array}{l}\text { BL-6M paired } \\
\text { P values }\end{array}$} \\
\hline & $\mathbf{n}$ & Median \pm IQR & & & \\
\hline \multicolumn{6}{|c|}{ Peripheral capillary non-perfusion } \\
\hline \multicolumn{6}{|l|}{ Global } \\
\hline Inactive PDR at 6M & 23 & $26 \%-50 \%(2.0 \pm 2.3)$ & $26 \%-50 \%(2.0 \pm 1.6)$ & $0.3 \pm 0.9$ & 0.38 \\
\hline Active PDR at 6M & 10 & $51 \%-75 \%(3.3 \pm 1.3)$ & $51 \%-75 \%(2.9 \pm 1.3)$ & $-0.4 \pm 1.9$ & 0.87 \\
\hline$P$ values & & $0.03^{*}$ & $0.03^{*}$ & 0.79 & \\
\hline \multicolumn{6}{|l|}{ Oximetry, \% } \\
\hline \multicolumn{6}{|l|}{ Arteriolar } \\
\hline Inactive PDR at 6M & 23 & $96.7 \pm 5.6$ & $96.8 \pm 4.7$ & $-0.1 \pm 5.1$ & 0.92 \\
\hline Active PDR at 6M & 10 & $93.4 \pm 6.4$ & $93.3 \pm 7.2$ & $-1.7 \pm 2.5$ & $0.02 *$ \\
\hline$P$ values & & 0.45 & 0.09 & 0.06 & \\
\hline \multicolumn{6}{|l|}{ Venular } \\
\hline Inactive PDR at $6 \mathrm{M}$ & 23 & $67.2 \pm 12.1$ & $66.2 \pm 8.3$ & $-1.1 \pm 15.8$ & 0.69 \\
\hline Active PDR at 6M & 10 & $63.6 \pm 14.8$ & $68.7 \pm 14.7$ & $1.2 \pm 5.9$ & 0.16 \\
\hline$P$ values & & 0.61 & 0.66 & 0.27 & \\
\hline
\end{tabular}

Retinal oxygen saturation and peripheral capillary non-perfusion (CNP) at baseline and follow-up month 6 . Groups are compared as to inactive and active PDR at the specific time point. Peripheral CNP: $0-0.5$ (close to no CNP), 1\%-25\% (>0.5-1.5), 26\%-50\% (>1.5-2.5), 51\%-75\% (>2.5-3.5), 76\%-100\% (>3.5-4.0). 6M data are corrected for rescue panretinal laser photocoagulation given to patients with active PDR at $3 M(n=2)$.

${ }^{*}$ Statistically significant.

BL, baseline; CNP, capillary non-perfusion; PDR, proliferative diabetic retinopathy; $6 \mathrm{M}, 6$ months' follow-up. 
in the retinal vascular oxygen saturation have been reported. One study found lower venular oxygen saturation in patients earlier treated with PRP, ${ }^{14}$ and another study reported that the arteriolar oxygen saturation was seen to decrease $3 \mathrm{M}$ after PRP treatment, ${ }^{27}$ although these studies did not test responder versus non-responders. The results found in our study, although statistically significant, were not found to be clinical relevant.

The findings of this study should be interpreted in the context of the following limitations. Although adjustment of the UWFA image contrast was possible, a trend towards overestimation of CNP areas next to leaky areas may occur because of the sharp change in contrast colour. The moderated PCNP scale has a tendency to underestimate the degree of global PCNP; this was done deliberately in order to counterbalance the potential overestimation of CNP areas due to the mentioned contrast colour issue. Furthermore, grading UWFA images was challenging, especially in the superior and inferior quadrants, and therefore the far periphery in these quadrants may be under-represented because of covering eyelids.

Detecting predictive markers of disease activity has proven difficult, and no preoperative markers have to our knowledge been identified. Taking the DRS and ETDRS study into account, the reduction in risk of severe visual loss was found to be $>50 \%$ after standard PRP treatment, still leaving almost half of the treated patients at risk. The possible predictive power of the area of PCNP gives food to thought. Standard PRP treatment was in this study found to be insufficient when dealing with larger retinal areas of PCNP in patients with PDR. An individualised treatment approach might be preferable. This might be achieved by additional PRP at an early stage, more intense follow-up during the first 6 months after PRP and maybe a lower threshold for vitrectomy or intravitreal anti-vascular endothelial growth factor intervention in severe cases of unsuccessful PRP treatment.

In summary, in patients with PDR, after PRP treatment, those with active PDR at $6 \mathrm{M}$ had larger areas of PCNP at BL as compared with those who have inactive PDR. Thus, PCNP may be used as a predictive marker at BL for PDR activity after PRP treatment, paving the possibility for individually titrated PRP treatment.

Contributors JG conceived the aims and overall design of the study. TLT acquired the data and did the writing of the different sections, tables and figures. JG and TLT did the literature search and statistical analyses. All authors were involved in the study design, data analyses, data interpretation and revision of the paper. TLT and JG had access to the full raw dataset. The corresponding author had the final responsibility to submit for publication.

Funding This study was funded by The Region of Southern Denmark, The Velux Foundation.

\section{Competing Interests None declared.}

Patient consent Obtained.

Ethics approval Regional Scientific Ethics Committee (ID S-20140046).

Provenance and peer review Not commissioned; externally peer reviewed.

\section{REFERENCES}

1 Wong TY, Cheung CM, Larsen M, et al. Diabetic retinopathy. Nat Rev Dis Primers 2016;2:16012.

2 Grauslund J, Green A, Sjølie AK. Blindness in a 25-year follow-up of a populationbased cohort of Danish type 1 diabetic patients. Ophthalmology 2009;116:2170-4

3 Klein R, Lee KE, Gangnon RE, et al. The 25-year incidence of visual impairment in type 1 diabetes mellitus the Wisconsin epidemiologic study of diabetic retinopathy. Ophthalmology 2010;117:63-70.

4 Grauslund J, Green A, Sjølie AK. Prevalence and 25 year incidence of proliferative retinopathy among Danish type 1 diabetic patients. Diabetologia 2009:52:1829-35.

5 DRS. Preliminary report on effects of photocoagulation therapy. The Diabetic Retinopathy Study Research Group. Am J Ophthalmol 1976;81:383-96.

6 Wessel MM, Aaker GD, Parlitsis G, et al. Ultra-wide-field angiography improves the detection and classification of diabetic retinopathy. Retina 2012;32:785-91.

7 Shoughy SS, Arevalo JF, Kozak I. Update on wide- and ultra-widefield retinal imaging. Indian J Ophthalmol 2015;63:575-81.

8 Silva PS, Cavallerano JD, Haddad NM, et al. Peripheral lesions identified on ultrawide field imaging predict increased risk of diabetic retinopathy progression over 4 years. Ophthalmology 2015;122:949-56.

9 Silva PS, Dela Cruz AJ, Ledesma MG, et al. Diabetic retinopathy severity and peripheral lesions are associated with nonperfusion on ultrawide field angiography. Ophthalmology 2015;122:2465-72.

10 Shimizu K, Kobayashi Y, Muraoka K. Midperipheral fundus involvement in diabetic retinopathy. Ophthalmology 1981;88:601-12.

11 Jørgensen CM, Hardarson SH, Bek T. The oxygen saturation in retinal vessels from diabetic patients depends on the severity and type of vision-threatening retinopathy. Acta Ophthalmol 2014;92:34-9.

12 Khoobehi B, Firn K, Thompson $\mathrm{H}$, et al. Retinal arterial and venous oxygen saturation is altered in diabetic patients. Invest Ophthalmol Vis Sci 2013;54:7103-6.

13 Hardarson SH, Stefánsson E. Retinal oxygen saturation is altered in diabetic retinopathy. Br J Ophthalmol 2012;96:560-3.

14 Hammer M, Vilser W, Riemer T, et al. Diabetic patients with retinopathy show increased retinal venous oxygen saturation. Graefes Arch Clin Exp Ophthalmol 2009;247:1025-30.

15 Rilvén S, Torp TL, Grauslund J. Retinal oximetry in patients with ischaemic retinal diseases. Acta Ophthalmol 2017;95:119-127.

16 Armstrong RA. Statistical guidelines for the analysis of data obtained from one or both eyes. Ophthalmic Physiol Opt 2013;33:7-14.

17 Torp TL, Kawasaki R, Wong TY, et al. Changes in retinal venular oxygen saturation predict activity of proliferative diabetic retinopathy 3 months after panretinal photocoagulation. Br J Ophthalmol 2018;102:383-7.

18 Torp TL, Kawasaki R, Wong TY, et al. Temporal changes in retinal vascular parameters associated with successful panretinal photocoagulation in proliferative diabetic retinopathy: a prospective clinical interventional study. Acta Ophthalmol 2017.

19 MacCormick IJ, Maude RJ, Beare NA, et al. Grading fluorescein angiograms in malaria retinopathy. Malar J 2015:14:367.

20 Jørgensen CM, Bek T. Lack of differences in the regional variation of oxygen saturation in larger retinal vessels in diabetic maculopathy and proliferative diabetic retinopathy. Br J Ophthalmol 2017;101:752-757.

21 Geirsdottir A, Palsson 0, Hardarson SH, et al. Retinal vessel oxygen saturation in healthy individuals. Invest Ophthalmol Vis Sci 2012;53:5433-42.

22 Landis JR, Koch GG. The measurement of observer agreement for categorical data. Biometrics 1977:33:159-74.

23 Guduru A, Martz TG, Waters A, et al. Oxygen saturation of retinal vessels in all stages of diabetic retinopathy and correlation to ultra-wide field fluorescein angiography. Invest Ophthalmol Vis Sci 2016;57:5278-84.

24 Bressler NM, Beck RW, Ferris FL. Panretinal photocoagulation for proliferative diabetic retinopathy. N Engl J Med 2011;365:1520-6.

25 Tamura T, Tamura M. Perifoveal capillary network and visual prognosis in diabetic retinopathy. Ophthalmologica 1982;185:141-6.

26 Takahashi K, Kishi S, Muraoka K, et al. Reperfusion of occluded capillary beds in diabetic retinopathy. Am J Ophthalmol 1998;126:791-7.

27 Jørgensen C, Bek T. Increasing oxygen saturation in larger retinal vessels after photocoagulation for diabetic retinopathy. Invest Ophthalmol Vis Sci 2014;55:5365-9. 